\title{
Polysèmes
}

Revue d'études intertextuelles et intermédiales

\section{Dans la nuit mystique : Black Dogs de Ian McEwan}

The Dark Night of the Soul in Black Dogs by Ian McEwan

\section{Marie Laniel}

\section{(2) OpenEdition \\ Journals}

Édition électronique

URL : http://journals.openedition.org/polysemes/412

DOI : $10.4000 /$ polysemes. 412

ISSN : 2496-4212

Éditeur

SAIT

\section{Référence électronique}

Marie Laniel, « Dans la nuit mystique : Black Dogs de lan McEwan », Polysèmes [En ligne], 13 | 2015, mis en ligne le 26 août 2016, consulté le 19 avril 2019. URL : http://journals.openedition.org/ polysemes/412 ; DOI : 10.4000/polysemes.412

Ce document a été généré automatiquement le 19 avril 2019.

Polysèmes 


\title{
Dans la nuit mystique : Black Dogs de Ian McEwan
}

\author{
The Dark Night of the Soul in Black Dogs by Ian McEwan
}

\section{Marie Laniel}

1 Fascinante exploration du mal, plongée dans la «nuit obscure » de l'âme humaine, Black Dogs (1992), le cinquième roman de Ian McEwan, interroge le pouvoir de l'écriture à conjurer le retour des spectres dans le monde postlapsaire issu de la Shoah et à « exhiber l'abject sans se confondre avec lui $»^{1}$. Plus encore que dans ses précédents romans, la mise en crise du récit biographique et historique s'accompagne d'une exploration aux limites $\mathrm{du}$ dicible, confrontant la narration à son Autre absolu. Pour approcher l'innommable, c'est dans le mysticisme que l'écriture semble trouver la voie d'un autre mode d'accès à la vérité et la trace de ses affinités secrètes avec la nuit. Mais l'expérience mystique cruciale vers laquelle le récit remonte lentement, et que le narrateur entreprend de reconstituer, reste elle-même un point obscur, objet inaccessible, diffracté par plusieurs récits enchâssés et fragmentaires. L'épreuve de l'inhumain condamne-t-elle l'écriture au silence en la confrontant à sa propre impossibilité? ou manifeste-t-elle une puissance de défiguration qui lui est essentielle?

Histoire d'une venue à l'écriture, celle du narrateur, Black Dogs trouve son origine dans le constat d'une absence. Orphelin à huit ans, Jeremy n'écrit pas depuis une position stable d'autorité, mais sous le coup d'une perte irréparable. Dès la "préface» du roman, la construction d'une posture énonciative est pour lui intimement liée à un sentiment d'abandon, une expérience de l'absence et de la nuit («the blackness, the hollow feeling of unbelonging $»^{2}$ ), qu'elle ne vient pas combler, mais avec laquelle elle "compose», et qui devient la matière du texte. Pâle adolescent usurpant l'affection des parents des autres, condamné à habiter toujours la zone d'ombre entre jeunesse et maturité, Jeremy n'a pas d'histoire. Aussi, lorsqu'il trouve finalement auprès de ses beaux-parents, June et Bernard Tremaine, une famille de substitution, le jeune homme s'approprie leur « roman familial ", pour en faire la matière de son propre récit ${ }^{3}$. Dès l'origine, ce projet d'écriture 
fondé sur un récit «de substitution » prend donc la mesure d'une absence, celle d'une histoire personnelle, et compose avec un invisible essentiel.

Compilation de manuscrits écrits successivement par Jeremy, Black Dogs se présente comme une série d'esquisses préparatoires à la rédaction des mémoires de June Tremaine, racontant dans un ordre qui n'est pas chronologique plusieurs épisodes significatifs de la vie de Jeremy et de ses beaux-parents : le premier chapitre, consacré aux dernières années de la vie de June, contient son témoignage sur sa conversion au mysticisme ("Wiltshire»), le deuxième se situe à Berlin au moment de la chute du Mur ( Berlin »), le chapitre suivant raconte la visite par Jeremy et son épouse Jenny du camp de concentration de Majdanek en Pologne, puis le parcours initiatique de Jeremy dans le Languedoc sur les traces de June et Bernard (« Majdanek, Les Salces and St Maurice de Navacelles $1989 »)$, et le récit remonte lentement vers la scène originelle racontée dans le dernier chapitre («St Maurice de Navacelles $1946 »)$ : la conversion de June au mysticisme après sa confrontation violente avec deux chiens noirs :

June came to God in 1946 through an encounter with evil in the form of two dogs. (Bernard found this construction of the event almost too embarrassing to discuss.) A malign principle, a force in human affairs that periodically advances to dominate and destroy the lives of individuals or nations, then retreats to await the next occasion; it was a short step from this to a luminous countervailing spirit, benign and all-powerful, residing within and accessible to us all; perhaps not so much a step as a simultaneous recognition. ${ }^{4}$

Fasciné par la puissance dévorante de cette image, le narrateur se laisse entraîner sur les traces des chiens noirs vers sa propre violence originaire, son propre «cœur de ténèbres", en un parcours qui ne peut se faire qu'à rebours de l'ordre chronologique, mais aussi à rebours de la maîtrise des signes et du langage.

4 Tout commence donc en 1946, lorsque, jeunes mariés dans une Europe qui sort à peine de la Guerre, forts de leur adhésion récente au Parti Communiste, Bernard et June Tremaine partent en voyage de noces dans le Languedoc. Lors d'une randonnée dans les Causses du Larzac, alors que Bernard, entomologiste, se laisse absorber par la contemplation d'une colonie de chenilles processionnaires, June s'engage seule dans un chemin creux, et se retrouve face à deux créatures, qu'elle prend d'abord pour des ânes, mais qui se révèlent être deux mastiffs noirs d'une taille monstrueuse ( black dogs of an unnatural size $»^{5}$ ). Comme dans Faust, le diable lui apparait sous la forme d'un chien avant de révéler son vrai visage à l'issue d'une métamorphose :

The creatures that blocked the path seventy yards ahead were only dogs in outline. In size they resembled mythical beasts. The suddenness of them, the anomaly, prompted the thought of a message in dumbshow, an allegory for her decipherment alone. She had a confusing thought of something medieval, of a tableau both formal and terrifying. At this distance the animals appeared to be grazing quietly. They emanated meaning. ${ }^{6}$

"Anomalie » échappant à l'ordre naturel, les chiens se situent dans un entre-deux, territoire par excellence du fantastique : défiant les frontières entre les espèces et entre les règnes, ils évoquent l'hybridité mythique de la chimère.

5 À l'origine d'un violent sentiment d'horreur, la présence incongrue des chiens s'accompagne d'un effet de saturation signifiante («message in dumbshow », « allegory », " decipherment», " emanated meaning »), le sentiment qu'un sens caché se manifeste, mais excède toute interprétation. C'est la noirceur des chiens, un invisible lié à une saturation du visible, qui concentre cette charge signifiante indéchiffrable : 
Their blackness, that they should both be black, that they belonged together and were without an owner made her think of apparitions. June did not believe in such things. She was drawn to the idea now because the creatures were familiar. They were emblems of the menace she had felt, they were the embodiment of the nameless, unreasonable, unmentionable disquiet she had experienced that morning. ${ }^{7}$

Ce que June reconnaît et identifie à cet instant ("the creatures were familiar »), c'est le sentiment de peur indéfinissable qu'elle a ressenti en s'engageant dans cette promenade le matin même, le pressentiment qu'une obscurité essentielle se cachait au sein de la lumière aveuglante du Midi (« The empty air, suffused with sunlight, seemed to contain a darkness just beyond the reach of vision $\left.»^{8}\right)$. Emblèmes de cette peur primitive irreprésentable («emblems of the menace»), les chiens sont le signe opaque d'une terreur sans nom qui excède tout objet ("nameless », « unreasonable », « unmentionable»). Instaurant une disjonction entre le visible et le dicible, les chiens constituent "un hiéroglyphe qui condense toutes les peurs ", un "conglomérat de peur, de privation et de frustration sans nom qui sont à proprement parler de l'innommable ${ }^{9}$. Leur pouvoir occulte tient précisément à ce qu'ils ne renvoient pas à un objet assignable, mais ont « la logique d'une métaphore et d'une hallucination » ${ }^{10}$.

De nature proprement mystique, puisque liée à la manifestation d'un sens caché indicible, la confrontation physique de June avec les chiens entraîne une révélation soudaine de la présence du divin, elle aussi de nature indicible, inspirée par le motif de la «nuit obscure» :

She tried to find the space within her for the presence of God and thought she discerned the faintest of outlines, a significant emptiness she had never noticed before, at the back of her skull. It seemed to lift and flow upwards and outwards, streaming suddenly into an oval penumbra many feet high, an envelope of rippling energy, or, as she tried to explain it later, of "coloured invisible light" that surrounded her and contained her. ${ }^{11}$

La manifestation du divin sur le mode de la "vacuité signifiante " «significant emptiness ») et de la «lumière invisible» (« invisible light ») associe immédiatement cette révélation à certains aspects de l'expérience mystique caractéristiques de la «théologie négative » de Grégoire de Nysse, Denys l'Aréopagite, Maître Eckhart ou Jean de la Croix. Selon les principes de la théologie négative, la seule connaissance possible du divin s'établit au-delà du sensible et de l'intelligible, et passe par l'épreuve de la « nuit du sens » et de la «nuit de l'esprit ${ }^{12}$. Ainsi, selon Denys l'Aréopagite, le divin est un «mystère indicible et inconnaissable, situé au-delà de tout ${ }^{13}$, et c'est uniquement en renonçant à toute appréhension sensible ou intellectuelle de Dieu que l'on peut être initié aux «secrets de cette radieuse et resplendissante Ténèbre, en sa totale obscurité absolument intangible et invisible, Ténèbre qui comble d'indicibles splendeurs les intelligences qui savent clore leurs yeux $»^{14}$. Maître Eckhart présente le Divin comme un " pur néant », « c'est-à-dire l'opposé de tout ce qui est et peut être connu » ${ }^{15}$. Inspiré par la théologie mystique de Denys l'Aréopagite, Jean de la Croix décrit la foi comme une « contemplation obscure $»^{16}$ mais certaine du divin par-delà les sens et l'intellect, par-delà l'imagination et la faculté discursive. Il a recours à l'image de la «nuit obscure » («nuit des sens » et "nuit de l'esprit») pour désigner l'épreuve des ténèbres sensibles puis spirituelles que l'âme doit endurer avant d'être éblouie par la lumière divine, le dépouillement radical nécessaire à l'union avec Dieu, et la révélation d'une "présence intense au sein de l'absence $»^{17}$. 
7 Expérience simultanée du mal absolu et de l'existence du divin, la rencontre avec les chiens noirs constitue à la fois un point d'opacité qui excède toute signification et une source d'éblouissement qui fonde l'écriture. À ce titre, les chiens noirs trouvent naturellement leur place dans le bestiaire mystique sous la forme de l'oxymore, ce "monstre ", capable de "creuser le langage en direction d'un indicible", cet "être linguistique étrange », "dont les deux moitiés appartiennent à des ordres différents et dont la tête, invisible, habite un autre espace $»^{18}$ :

L'oxymoron appartient à la catégorie des «métasémèmes » qui renvoient à un audelà du langage, comme le fait le démonstratif. C'est un déictique : il montre ce qu'il ne dit pas. La combinaison des deux termes se substitue à l'existence d'un troisième et le pose comme absent. Elle crée un trou dans le langage. Elle y taille la place d'un indicible. C'est du langage qui vise un non-langage. [...] Dans un monde supposé tout entier écrit et parlé, lexicalisable donc, il ouvre le vide d'un innommable, il pointe une absence de correspondance entre les choses et les mots. ${ }^{19}$

Incarnation d'une tension oxymorique qui travaille le langage de l'intérieur, les chiens noirs substituent au principe de la nomination une logique de la monstration. Situés à la «jointure de deux mondes $»^{20}$, ils déstabilisent le fonctionnement naturel du signe qui ne fait plus sens de façon transitive, dans sa transparence signifiante, mais par son opacité même : c'est la noirceur des chiens qui est signifiante. Selon les termes de Michel de Certeau, le langage mystique est ainsi "un producteur de secret», qui repose sur l'opacification du signe, et «tend à faire disparaître la chose signifiée au bénéfice du signifiant ${ }^{21}$. Alors que « dans la théorie classique, le signe est biface : il représente une chose (reconnaissable 'à travers' lui) et il a une réalité propre (qui constitue son épaisseur) », " par le premier aspect, il est transparent ; par le second, il est opaque », les poèmes mystiques « retirent [aux] mot[s] leur valeur désignative, et, de ce fait, leur transparence de signes ${ }^{22}$, en mettant l'accent sur la partie opaque du signe en lui-même. Loin de se réduire à un symbole, symbole du mal ou d'un accès à l'au-delà, loin d'être le signe d'autre chose, les chiens noirs représentent l'opacité essentielle du signe, échappent à l'expérience de la parole transitive et renvoient à « un fantastique de la signification $»^{23}$. Au sein de l'ordre sym-bolique, « fiction productrice d'union », les chiens noirs introduisent la disjonction $\mathrm{du}$ dia-bolique, "dissuasion du symbolique par l'innommable de cette chose $»^{24}$, et manifestent une puissance dévorante de défiguration du langage.

9 Si elle échappe finalement à la mort et met les chiens noirs en fuite, cette expérience de l'horreur sacrée conduit June à renoncer au monde (et à son idéal communiste), pour vivre en recluse dans une bergerie perdue dans le Larzac, dont elle fait l'acquisition peu de temps après sa confrontation avec les chiens, et où elle compose des traités mystiques, Mystical Grace: Selected Writings of St Teresa of Avila et Ten Meditations. Dans la tradition mystique, la méditation requiert la délimitation d'un espace mental, d'un « champ propre de l'allocution ${ }^{25}$ («Le Verbe [...] doit naître dans le vide qui l'attend ${ }^{26}$ ), de « lieux énonciatifs $\aleph^{27}$ propices au dialogue avec Dieu, au surgissement de la voix. Dans Le Château intérieur (1577), Sainte Thérèse d'Avila compare l'âme, transparente à elle-même, à « un château, fait d'un seul diamant ou d'un cristal parfaitement limpide », " qui renferme de nombreuses demeures " $^{28}$. "Château intérieur ", " château de cristal ", la bergerie où June s'est retirée après avoir quitté Bernard est un lieu qui échappe à l'expérience transitive de la parole sociale.

10 Au fil des ans, la puissance de défiguration des chiens noirs fait peu à peu son œuvre sur le visage de June. Face à la faillite du langage, le corps prend le relais de la parole pour 
traduire cette expérience ineffable. Peu de temps avant sa mort, alors que Jeremy tente d'obtenir d'elle une version cohérente des événements, c'est le visage de June, plus que son récit erratique et fragmenté, qui témoigne de la violence de cette confrontation. Le visage autrefois lisse de la jeune fille s'est allongé et creusé de sillons formant une arborescence ("wrinkle tree»), emblème de cette métamorphose radicale, «a metempsychosis mapped in the transformation of her face $»^{29}$. À l'image de sa réclusion volontaire, ses traits se sont repliés sur eux-mêmes : "years of solitude and reflection distended the features, then folded them in on themselves $»^{30}$. Comparant une photographie de jeunesse au masque sépulcral de ses dernières années, Jeremy tente en vain de déchiffrer les marques que l'expérience de cette horreur sacrée a laissées sur son visage, autant de «messages illisibles » tracés sur « un corps transformé en emblème ou en mémorial $»^{31}$ :

In repose her face had a chiselled, sepulchral look; it was a statue, a mask carved by a shaman to keep at bay the evil spirit.

In this last there may have been some simple truth. She might have grown her face to accommodate her conviction that she had confronted and been tested by a symbolic form of evil. "No, you clot. Not symbolic!" I hear her correcting me. "Literal, anecdotal, true. Don't you know, I was nearly killed!" ${ }_{32}$

Devenu corps mystique, le corps de June « cesse d'être transparent au sens, il s'opacifie, et devient la scène muette d'un 'je ne sais quoi' qui l'altère $»^{33}$. Devenu emblème (à l'image des deux chiens monstrueux), son visage n'est plus le lieu de l'expression du sens, mais celui de la «monstration » d'un innommable.

Rongé par une forme de leucémie incurable, le corps de June échappe désormais à la logique du sens pour obéir à la seule logique du symptôme, ce «langage déclarant forfait», selon Julia Kristeva, qui «structure dans le corps un étranger inassimilable, monstre, tumeur et cancer $~_{34}$. Tout en dévorant son corps, les chiens noirs s'immiscent dans la vie psychique de June, et c'est d'ailleurs sur le récit suspendu d'un rêve récurrent que s'achève sa dernière entrevue avec Jeremy :

It was in my notebook, the short, unvarying, pre-sleep dream that had haunted her for forty years: two dogs are running down a path into the Gorge. The larger leaves a trail of blood, easily visible on the white stones. June knows that the mayor of a nearby village has not sent out his men to track the animals down. They descend into the shadow cast by the high cliffs, down into the thickets, and up the other side. She sees them again, across the Gorge, heading into the mountains, and even though they are going far away from her, this is the moment of terror that jolts her; she knows they will return..$^{35}$

La rémanence visuelle des chiens noirs sous forme d'hallucination manifeste ce déplacement du signe inassimilable par la parole, venu coloniser le corps et le psychisme : "You need to remember that it comes when I'm still half-awake. I actually see them, Jeremy $»^{36}$. Imprimée sur la rétine, l'image des chiens est cette "métaphore qui s'est trompée de lieu, quittant le langage pour la pulsion et la vision $»^{37}$. Après la mort de June, c'est à Jeremy qu'il revient de suivre la trace des chiens, de traquer cette "image " inassimilable, ce « signifiant » fuyant, pour tenter de le réintégrer à l'ordre du dicible.

Or, loin de rendre lisible cette expérience de l'inhumain, le récit livré par Jeremy en redouble et diffracte toutes les apories. Figure du biographe, cherchant à saisir la vie d'une disparue, elle-même guidée par l'invisible, le narrateur se retrouve dans la posture inconfortable de "l'historien des mystiques ", " appelé comme eux à dire l'autre ", selon Michel de Certeau, à "redouble[r] leur expérience en l'étudiant », puisqu' " un exercice d'absence définit à la fois l'opération par laquelle il produit son texte et celle qui a 
construit le leur $\aleph^{38}$. Condamné à se situer dans un entre-deux, Jeremy refuse de trancher entre les réactions contradictoires de ses beaux-parents face à l'horreur de la Shoah : le refuge dans la «nuit mystique » pour June ou les promesses du « Grand Soir », la marche de l'Histoire guidée par la Raison, pour Bernard : « Rationalist and mystic, commissar and yogi, joiner and abstainer, scientist and intuitionist, Bernard and June are the extremities, the twin poles along whose slippery axis my own unbelief slithers and never comes to rest $\aleph^{39}$. Pourtant, précisément parce qu'il est condamné à rester dans l'entredeux de cette position indécidable, l'expérience narrative de Jeremy est plus proche de celle de June que de celle de Bernard ${ }^{40}$.

Le souvenir de l'expérience cruciale ayant disparu avec June, Jeremy ne peut que tenter de saisir le reflet déformé des "chiens noirs", qui resurgissent non pas sous la forme d'une entité physique, mais sous la forme d'un signifiant fuyant, frayant son chemin à travers l'Histoire de l'humanité et déstabilisant toute interprétation. Dans le dernier chapitre du roman, il est suggéré que les deux chiens vaincus par June avaient été dressés par la Gestapo pendant l'Occupation pour violer les prisonnières. Deux ans après la mort de June, au moment de la chute du Mur de Berlin, Jeremy et Bernard se retrouvent tous deux dans la capitale réunifiée pour assister au passage à l'Ouest de milliers d'EstAllemands. Alors que les deux hommes reviennent sur les événements qui conduisirent à la conversion de June quarante ans plus tôt, l'horreur de l'Histoire passée semble prête à resurgir : le fil de leurs déambulations les entraîne devant les anciens quartiers généraux de la Gestapo, bientôt transformés en musée, «Gestapo crimes neutralised by archaeology $»^{41}$. Au milieu des célébrations, les mouvements de la foule trahissent la présence latente d'instincts animaux - "A crowd is a slow, stupid creature, far less intelligent than any one of its members. This one was prepared to stand all night, with the patience of a dog $[. ..] »^{42}-$, et c'est en effet dans l'anonymat de la foule que les chiens noirs font retour, sous la forme d'une bande de néo-nazis qui attaquent un manifestant communiste isolé. Alors qu'il tente de s'interposer, Bernard est roué de coups et n'échappe à la mort que grâce à l'intervention d'une jeune femme, ressemblant étrangement à June.

Si Jeremy voit dans l'intervention de Grete la manifestation du pouvoir apotropaïque de June, Bernard tente de réintégrer cet épisode à une lecture rationnelle de l'Histoire, réduisant les chiens noirs à un symbole, celui d'une crise de la civilisation, «cultural depression $»$ :

"Bernard, I wanted to ask you something about June's dogs..."

He interrupted me. "For the life and times? I'll tell you something. You can forget all that nonsense about 'face to face with evil'. Religious cant. But, you know, I was the one who told her about Churchill's black dog. You remember? The name he gave to the depressions he used to get from time to time. I think he pinched the expression from Samuel Johnson. So June's idea was that if one dog was a personal depression, two dogs were a kind of cultural depression, civilisation's worst moods. Not bad, really. I've often made use of it. It went through my mind at Checkpoint Charlie." 43

La réaction de Bernard est celle de l'historien, qui reconnaît la puissance métaphorique de l'image ou du mythe, mais refuse de se confronter à sa noirceur radicale. Contrairement au mystique toujours dans l'attente du retour de l'Absent, l'historien cherche à résorber l'étrangeté de l'événement dans une logique supérieure : «L'historien 'calme' les morts et lutte contre la violence en produisant une raison des choses (une 
'explication') qui surmonte leur désordre et certifie des permanences; le mystique, en fondant l'existence sur son rapport même avec ce qui lui échappe $»^{44}$ :

In Black Dogs, June believes in evil in these terms, and her husband Bernard does not. But he knows it's a potent idea. It's a useful way of talking about a side of human nature, and it's metaphorically rich, and for that reason, hard to live without. Harder to live without evil, it would seem, than without God. ${ }^{45}$

En résorbant l'image des « chiens noirs » dans une symbolique historique, celle de la crise de la civilisation, Bernard lui retire sa dimension aporétique. La voix narrative conserve au contraire à l'image toute son ambiguïté en rapprochant les chiens noirs de la meute des assaillants de Jeremy («The kids [...] bunched up in a pack, breathing heavily, heads and tongues lolling in bemusement at this beanpole, this scarecrow in a coat who stood in their way $\left.{ }^{46}\right)$, mais en suggérant également une parenté entre les créatures et les deux jeunes filles, aperçues plus tôt dans la foule, dont l'une porte secours à Bernard (« Two girls in black leather jackets", " Their hair was dyed black. The identical pony-tails that swung behind them completed a passing reference to the fifties $»^{47}$ ). Sans s'engager comme June dans la voie du mysticisme, mais sans rallier la position sceptique de son beau-père, Jeremy suggère que le pouvoir de défiguration des chiens noirs est aussi à l'œuvre dans l'Histoire, porteur d'un indicible, celui de l'humanité se retournant contre elle-même.

L'épisode berlinois fait en effet resurgir dans la mémoire de Jeremy un souvenir enfoui : la visite avec son épouse Jenny du camp de concentration de Majdanek en Pologne. Dans l'enceinte du camp, la mince pellicule de civilisation qui semble recouvrir l'horreur éclate et laisse surgir l'innommable : «transform[ing] Majdanek for me in an instant from a monument, an honourable civic defiance of oblivion, to a disease of the imagination and a living peril, a barely conscious connivance with evil $\aleph^{48}$. Loin de s'élever à la mémoire des victimes, le monument trahit une collusion avec l'horreur. Lorsqu'il évolue dans le camp, Jeremy est contraint d'adopter la position non des victimes, mais celle des bourreaux. La posture de touriste qui se confond avec la posture d'énonciation lui est insoutenable. C'est d'ailleurs devant le camp que Jeremy entend pour la première fois l'expression « the black dogs ${ }^{49}$ dans la bouche de Jenny, expression dont il apprend qu'elle appartient au jargon familial («Black dogs. It's a family phrase, from my mother $»^{50}$ ), mais sans que lui soit révélé ce que le signifiant recouvre réellement. Le signifiant opaque qui se glisse dans la langue commune acquiert une familiarité encore plus troublante et pose la question de la collusion de l'écriture avec le mal. Tout comme le camp de Majdanek, l'œuvre écrite qui veut faire monument a partie liée avec l'inhumain.

17 À mesure qu'il s'approprie le roman familial de June et Bernard, Jeremy se laisse peu à peu investir par des voix étrangères et éprouve lui aussi la collusion essentielle de l'écriture avec la nuit. Après son passage à Berlin avec Bernard, la dernière étape de son parcours le conduit tout d'abord dans la bergerie du Larzac, « château intérieur » de June, espace mental propice au retour des spectres :

I opened the front door and stood before a wall of darkness. Then I reached inside, up to a shelf where we tried to remember to keep a candle and a box of matches. There was nothing there. I stood and listened. Whatever sensible thing I told myself, I could not banish the thought that in a house where a woman had given herself for so many years to the contemplation of eternity, some delicate emanation, a gossamer web of consciousness inhered and was aware of me. ${ }^{51}$ de vacances vide et gagnée par les ténèbres évoque un autre texte majeur sur la 
figuration de l'absence, la section "Time Passes » de To the Lighthouse, où, selon Chantal Delourme, Virginia Woolf tente de figurer "l'autre nuit», une nuit qui ne serait pas l'absence du jour mais un invisible essentiel ${ }^{52}$. Au fond de cette "nuit essentielle ${ }^{53}$, Jeremy avance sans le savoir à la rencontre d'une bête tapie dans l'ombre : un scorpion logé sur un panneau de bois, dissimulé par l'obscurité, dont les contours lui apparaissent comme « a blacker patch $»^{54}$, « [a] shapeless deeper black $»^{55}$, « a patch of extra blackness in the dark $»^{56}$. Plus qu'une bête tapie dans l'ombre, c'est vers "la dissimulation" incarnée que Jeremy semble progresser, non pas «ce que dissimule la nuit » ${ }^{57}$, mais « la dissimulation qui se révèle dans la nuit $»^{58}$, « la profondeur [qui] ne se révèle qu'en se dissimulant $»^{59}$. Cette scène qui préfigure pour Jeremy la découverte de sa propre bestialité révèle à la fois les affinités de l'homme avec la bête et celles de l'écriture avec la nuit :

Il y a toujours un moment où, dans la nuit, la bête doit entendre l'autre bête. C'est l' autre nuit. Cela n'est nullement terrifiant, cela ne dit rien d'extraordinaire - rien de commun avec les fantômes et les extases -, ce n'est qu'un susurrement imperceptible, un bruit qu'on distingue à peine du silence [...]. Ce que la bête pressent dans le lointain, cette chose monstrueuse qui vient éternellement à sa rencontre, qui y travaille éternellement, c'est elle-même, et si elle pouvait jamais se trouver en sa présence, ce qu'elle rencontrerait, c'est sa propre absence, c'est ellemême, mais elle devenue l'autre, qu'elle ne reconnaîtrait pas, qu'elle ne rencontrerait pas. ${ }^{60}$

En s'aventurant dans la maison familiale, Jeremy revit l'expérience de June: la confrontation avec un signe opaque, emblème d'une menace sans nom et hiéroglyphe de toutes les peurs. L'effacement des contours du scorpion et la déstabilisation des limites entre sujet et objet annonce la confrontation de Jeremy avec sa propre part d'ombre à l'Hôtel des Tilleuls.

19 L'ultime étape du parcours de Jeremy à rebours du temps et de l'itinéraire emprunté autrefois par June et Bernard le conduit de la bergerie aux Causses du Larzac. Alors qu'il pense conclure son mémoire, un événement relance l'écriture de façon inopinée, lorsque Jeremy fait la découverte de sa propre noirceur. Alors qu'il fait étape à Saint Maurice de Navacelles, dans l'hôtel fréquenté autrefois par June et Bernard, Jeremy prend la défense d'un enfant battu par ses parents et attaque le père avec un déchaînement de violence tel qu'une cliente doit intervenir. Possédé par les chiens noirs, Jeremy obéit à la même injonction que celle que June avait utilisée pour tenir les chiens en respect : « ça suffit !». Le narrateur franchit ainsi la «ligne d'ombre", "crossing the shadow line and going deeper where the sun never reaches $»^{61}$, la frontière ténue entre civilisation et barbarie.

Dans son analyse de la pulsion phobique, Julia Kristeva a montré que la peur de la dévoration est bien souvent le "signe inversé » d'une agressivité primaire qui ne peut s'exprimer. C'est parce que je ne peux nommer ma propre violence que je la transfère en l'inversant sur un animal dévorant («Ce n'est pas moi qui dévore, je suis dévoré par lui, un tiers, donc [il, un tiers] me dévore $»^{62}$. Ces processus de passivation qui représentent une étape fondamentale dans la constitution de la subjectivité, sont aussi, selon Julia Kristeva, au fondement de l'activité symbolique : "C'est seulement après cette inversion que le 'cheval' ou le 'chien' peut devenir la métaphore de ma bouche vide et incorporante qui me regarde, menaçante, du dehors $\aleph^{63}$. Si la phobie en tant que pathologie est liée à une faillite du système signifiant, une migration de la métaphore non verbalisable vers le psychisme, elle révèle cependant un mécanisme fondamental de toute activité symbolique : l'incapacité à reconnaître le mal en soi serait à l'origine de la capacité du 
sujet à métaphoriser ${ }^{64}$. Déplacement de l'inhumain par une métaphore, l'activité symbolique aurait donc fondamentalement partie liée avec la peur, la perversion, la violence : «Harder to live without evil, it would seem, than without God »"

Si l'activité symbolique trouve son origine dans une violence primaire, les " chiens noirs " ne seraient pas seulement le signe opaque d'une peur sans nom, mais le signifiant de l'activité symbolique elle-même, ce point aveugle de la représentation, trop profond et fondamental pour être appréhendé ou saisi en pleine lumière : cette violence primaire qui conduit à métaphoriser. Pour ne pas être anéanti par la puissance dévorante des chiens, Jeremy est en effet condamné à les faire siens, à les assimiler par l'écriture en un acte $\mathrm{d}^{\prime}$ " anthropophagie symbolique $»^{66}$ caractéristique de la parole poétique, de la relation d'Orphée avec les Ménades, selon Julia Kristeva. C'est en effet comme signifiant sublimé, mais insaisissable, objet toujours fuyant de l'écriture que les chiens apparaissent dans les dernières pages du roman ( $«$ But it is the black dogs I return to most often. They trouble me when I consider what happiness I owe them, especially when I allow myself to think of them, not as animals, but as spirit hounds, incarnations $»^{67}$ ). Et c'est sur le rêve obsédant de June, l'image des chiens noirs en fuite, que se conclut le récit :

June told me that throughout her life she sometimes used to see them, really see them, on the retina in the giddy seconds before sleep. They are running down the path into the Gorge of the Vis, the bigger one trailing blood on the white stones. They are crossing the shadow line and going deeper where the sun never reaches, and the amiable drunken mayor will not be sending his men in pursuit for the dogs are crossing the river at the dead of night, and forcing a way up the other side to cross the Causse; and as sleep rolls in they are receding from her, black stains in the grey of the dawn, fading as they move into the foothills of the mountains from where they will return to haunt us, somewhere in Europe, in another time. ${ }^{68}$

Saisi par Jeremy dans leur mouvement infini de fuite, les chiens noirs représentent ce signifiant fondamental sans objet qui happe l'écriture et l'entraîne aux confins de la visibilité. Reflet inversé de l'humain, fixé à jamais sur la rétine de June, ils sont cette image imprimée sur l'œil qui est au fondement même de toute tentative de représentation et qui entraîne l'écriture vers la découverte de son " pouvoir nocturne ». Fixant à jamais la course des chiens noirs dans le crépuscule, le texte atteint ce point sublime où, selon Julia Kristeva, «l'abject s'effondre dans l'éclatement du beau qui nous déborde $»^{69}$.

\section{BIBLIOGRAPHIE}

Blanchot, Maurice. L'Espace littéraire. Paris : Gallimard, 1955.

Certeau, Michel de. La Fable mystique :XVI ${ }^{e}-X V I I^{e}$ siècle. Paris : Gallimard, 1982.

Delourme, Chantal. « La figure, la nuit dans 'Time Passes' ». Études britanniques contemporaines numéro hors-série « Société d'Études Woolfiennes » (1997) : 119-134.

Jean de la Croix. Cuvres complètes : la nuit obscure. Paris : Éditions du Cerf, 1982.

Jossua, Jean-Pierre. Seul avec Dieu : l'aventure mystique. Paris : Gallimard, 1996. 
Kristeva, Julia. Pouvoirs de l'horreur. Paris : Seuil, 1980.

Kristeva, Julia. Soleil noir : dépression et mélancolie. Paris : Gallimard, 1987.

McEwan, Ian. Black Dogs (1992). London: Vintage, 1998.

Pedot, Richard. Perversions textuelles dans la fiction d'Ian McEwan. Paris : L'Harmattan, 1999.

Pedot, Richard. « Une narration en quête de son sujet : chemin de l'écriture et écriture du chemin dans Black Dogs de Ian McEwan ». Études britanniques contemporaines 11 (1997) : 67-75.

Pseudo-Denys. La Théologie mystique. Trad. Madeleine Cassingena. Paris : Migne, 1991.

Roberts, Ryan (ed.). Conversations with Ian McEwan. Jackson: Mississippi UP, 2010.

Thérèse d'Avila. CEuvres complètes. Vol. 1. Paris : Éditions du Cerf, 1995.

\section{NOTES}

1. Julia Kristeva, Pouvoirs de l'horreur, Paris : Seuil, 1980, 247.

2. Ian McEwan, Black Dogs (1992), London: Vintage, 1998, 10.

3. Richard Pedot décrit ce "passage de relais narratif " entre June et Jeremy dans le premier chapitre du roman (Perversions textuelles dans la fiction d'Ian McEwan, Paris : L'Harmattan, 1999, 264-265).

4. McEwan, op. cit., 19.

5. Ibid., 144.

6. Ibid., 144.

7. Ibid., 144-145.

8. Ibid., 141.

9. Kristeva, op. cit., 46.

10. Ibid., 46.

11. McEwan, op. cit., 149-150.

12. Jean de la Croix, Euvres complètes : la nuit obscure, Paris : Éditions du Cerf, 1982, 64.

13. Jean-Pierre Jossua, Seul avec Dieu : l'aventure mystique, Paris : Gallimard, 1996, 21.

14. Pseudo-Denys, La Théologie mystique, cité par Jean-Pierre Jossua, ibid., 164.

15. Jossua, op. cit., 64.

16. Jean de la Croix, op. cit., 31.

17. Jossua, op. cit., 87.

18. Michel de Certeau, La Fable mystique : XVI ${ }^{e}-X V I I^{e}$ siècle, Paris : Gallimard, 1982, 199.

19. Ibid., 198-199.

20. Ibid., 199.

21. Ibid., 201.

22. Ibid., 200-201.

23. Ibid., 83.

24. Ibid., 58.

25. Ibid., 221.

26. Ibid., 14.

27. Ibid., 227.

28. Thérèse d'Avila, Euvres complètes, vol. 1, Paris : Éditions du Cerf, 1995, 969.

29. McEwan, op. cit., 27.

30. Ibid., 28.

31. De Certeau, op. cit., 14. 
32. McEwan, op. cit., 28.

33. De Certeau, op. cit., 15.

34. Kristeva, op. cit., 19.

35. McEwan, op. cit., 61-62.

36. Ibid., 62.

37. Kristeva, op. cit., 47.

38. De Certeau, op. cit., 21.

39. McEwan, op. cit., 19.

40. Pedot, op. cit., 250.

41. McEwan, op. cit., 93.

42. Ibid., 88.

43. Ibid., 104.

44. De Certeau, op. cit., 21.

45. Propos de Ian McEwan recueillis par Ryan Roberts (Conversations with Ian McEwan, Jackson: Mississippi UP, 2010, 101).

46. McEwan, op. cit., 97.

47. Ibid., 83.

48. Ibid., 110 .

49. Ibid., 110

50. Ibid., 112

51. Ibid., 114

52. Voir l'article de Chantal Delourme, «La figure, la nuit dans 'Time Passes' », Études britanniques contemporaines numéro hors-série « Société d'Études Woolfiennes » (1997) : 119-134.

53. Maurice Blanchot, L'Espace littéraire, Paris : Gallimard, 1955, 222.

54. McEwan, op. cit., 114-115.

55. Ibid., 115 .

56. Ibid., 118 .

57. Blanchot, op. cit., 227.

58. Ibid., 225.

59. Ibid., 226 .

60. Ibid., 221

61. McEwan, op. cit., 174.

62. Kristeva, op. cit., 50 .

63. Ibid., 51.

64. « Parallèle à la constitution de la fonction signifiante, la phobie qui opère, elle aussi, sous le coup de la censure et du refoulement, déplace en inversant le signe (l'actif devient passif) avant de métaphoriser », ibid., 51.

65. Propos de Ian McEwan recueillis par Ryan Roberts, op. cit., 101.

66. Julia Kristeva, Soleil noir : dépression et mélancolie, Paris : Gallimard, 1987, 157.

67. McEwan, op. cit., 173.

68. Ibid., 173-174.

69. Kristeva, Pouvoirs de l'horreur, op. cit., 248. 


\section{RÉSUMÉS}

Fascinante exploration du mal, plongée dans la «nuit obscure » de l'âme humaine, Black Dogs (1992), le cinquième roman de Ian McEwan, interroge le pouvoir de l'écriture à conjurer le retour des spectres dans le monde postlapsaire issu de la Shoah et à « exhiber l'abject sans se confondre avec lui ». Plus encore que dans ses précédents romans, la mise en crise du récit biographique et historique s'accompagne d'une exploration aux limites du dicible, confrontant la narration à son Autre absolu. Pour approcher l'innommable, c'est dans le mysticisme que l'écriture semble trouver la voie d'un autre mode d'accès à la vérité et la trace de ses affinités secrètes avec la nuit. Mais l'expérience mystique cruciale vers laquelle le récit remonte lentement et que le narrateur entreprend de reconstituer reste elle-même un point obscur, objet inaccessible, diffracté par plusieurs récits enchâssés et fragmentaires. L'épreuve de l'inhumain condamne-t-elle l'écriture au silence en la confrontant à sa propre impossibilité ? Ou manifeste-t-elle une puissance de défiguration qui lui est essentielle? Si l'activité symbolique trouve son origine dans une violence primaire, alors les « chiens noirs " ne sont pas seulement le signe opaque d'une peur sans nom, mais le signifiant de l'activité symbolique elle-même, ce point aveugle de la représentation, trop profond et fondamental pour être appréhendé ou saisi en pleine lumière : cette violence primaire qui nous conduit à métaphoriser.

In Black Dogs (1992), his fifth novel, Ian McEwan probes the "dark night" of the human soul and questions the power of literature to lay to rest the ghosts of the past in a postlapsarian, postShoah world and to "expose the abject without being tainted by it". More than ever, the disruption of traditional biographical and historical narrative patterns leads to an exploration into the unspeakable and a confrontation with some radical form of Otherness. The encounter with evil in the shape of two black dogs can only be conveyed through a mystical form of experience, providing another access to truth and exposing the secret affinities between writing and mystical night. This crucial experience, however, remains inaccessible, diffracted by competing and fragmentary narratives. Does the confrontation with barbarism condemn literature to silence? Or does it convey a power of dis-figurement which is integral to it? If our need for symbols originates in primeval violence, then the "black dogs" are not only the opaque sign of unnameable fear, but the secret signifier of our own symbolic activity, a blind spot, too deep and fundamental to be seized in full light: the primeval violence which leads us to produce metaphors.

\section{INDEX}

oeuvrecitee Black Dogs

Mots-clés : obscurité, mysticisme, Saint Jean de la Croix, Sainte Thérèse d'Avila

Keywords : darkness, mysticism, Saint John of the Cross, Saint Teresa of Avila 


\section{AUTEURS}

\section{MARIE LANIEL}

Ancienne élève de l'École Normale Supérieure et agrégée d'anglais, Marie Laniel est Maître de Conférences à l'Université de Picardie - Jules Verne. Ses recherches portent sur les réécritures de l'intertexte victorien dans les essais et la fiction de Virginia Woolf, notamment ses relectures subversives de l'œuvre de Thomas Carlyle (« Revisiting a Great Man’s House: Virginia Woolf's Carlylean Pilgrimages »), Alfred Tennyson (" "The name escapes me”: Virginia Woolf's Dislocation of Patrilineal Memory in A Room of One's Own »), Matthew Arnold (« Virginia Woolf, lectrice de Matthew Arnold : la fortune littéraire du "scholar-gipsy" dans les essais et la fiction») et Leslie Stephen ("Généalogies de l'essai : de Leslie Stephen à Virginia Woolf»). 